\title{
Neonatal Formula Feeding Leads to Immunological Alterations in an Animal Model of Type 1 Diabetes
}

\author{
RICARDO A. CAICEDO, NAN LI, CLOTILDE DES ROBERT, PHILIP O. SCUMPIA, CHAD P. HUBSHER, \\ CLIVE H. WASSERFALL, DESMOND A. SCHATZ, MARK A. ATKINSON, AND JOSEF NEU \\ Departments of Pediatrics [R.A.C., N.L., C.R., P.O.S., C.P.H., D.A.S., J.N.] and Department of Pathology [C.H.W., M.A.A.], \\ University of Florida College of Medicine, Gainesville, Florida 32610
}

\begin{abstract}
Neonatal diet may influence the development of type 1 diabetes (T1D) in susceptible individuals through an intestinal mucosal inflammatory response, resulting in loss of self-tolerance. We tested the hypothesis that formula feeding during the neonatal period accelerates the development of T1D in diabetes-prone BioBreeding (BBDP) rats through regulation of $\mathrm{CD} 4+\mathrm{CD} 25+$ regulatory $\mathrm{T}$ lymphocytes $\left(\mathrm{T}_{\text {reg }}\right)$ and anti-inflammatory cytokines. BBDP rat pups fed rat milk substitute (RMS) via a "pup-in-the cup" system were compared with mother-fed (MF) rats. The spleen and thymus were analyzed for Foxp3-expressing CD4+/CD25+ T cells. Multiplex enzyme-linked immunosorbent assays (ELISAs) were performed to measure cytokine-induced neutrophil chemoattractant (CINC), tumor necrosis factor $\alpha$ (TNF- $\alpha$ ), interferon-gamma (IFN$\gamma$ ), interleukin (IL)-4, IL-10, and IL-18. Diabetes-free survival, time of disease onset, and $\mathrm{T}_{\text {reg }}$ /total $\mathrm{T}$ lymphocyte ratios were not different. MF pups had higher ileal CINC $(p<0.001)$ and IL-18 $(p=$ 0.002 ), but no differences in the liver. There were no differences in ileal cytokine concentrations of 75-d-old rats, but the formula-fed rats had greater liver TNF- $\alpha(p<0.001)$, IFN- $\gamma$, and IL-4 $(p<0.01)$ and lower IL-10 ( $p=0.002)$ compared with MF animals. Formula versus maternal milk altered the hepatic cytokine profile at $75 \mathrm{~d}$ toward an inflammatory pattern but did not result in altered $\mathrm{T}_{\text {reg }}$ cell frequencies or the development of T1D. (Pediatr Res 63: 303-307, 2008)
\end{abstract}

$\mathrm{T}$ yype 1 diabetes (T1D) is characterized by the autoimmune destruction of insulin-secreting beta cells, resulting in insulin deficiency and hyperglycemia in genetically susceptible individuals. While genetic predisposition is clearly a major component of T1D, interactions between the environment and the immune system are thought to weigh heavily in disease development (1). Indeed, with evidence pointing to the pathogenesis of T1D beginning very early in life or perhaps in utero (2), more attention has recently been directed toward environmental exposures early in infancy, particularly that of diet.

An underlying structural or developmental defect in the gastrointestinal tract may assist in the entry of dietary agents that have the potential to influence the T1D process $(3,4)$. In healthy individuals, the small intestine acts as a gatekeeper between the external environment and the internal organ systems of the body, allowing the absorption of nutrients and preventing the passage of potential antigens. During infancy,

Received May 31, 2007; accepted October 6, 2007.

Correspondence: Josef Neu, M.D., Department of Pediatrics, College of Medicine, University of Florida, PO Box 100296, Gainesville, FL 32610-0296; e-mail: neuj@peds.ufl.edu

Funded by NIH RO3 A142288. especially in the first 2 mo of life, increased intestinal permeability allows dietary antigens greater access to the resident immune cells of the lamina propria (5). Increased gut permeability has also been reported in T1D patients who do not have associated celiac disease (6).

BioBreeding diabetes-prone (BBDP) rats (Biomedical Research Models, Worcester, MA) are a well-studied model of T1D because at a predictable point in their life span (70-120 d), a large majority ( $>90 \%$ ) develops T1D manifested as overt glycosuria, hyperglycemia, and ketosis (7). The loss of tolerance that permits autoimmunity against the beta cell and the development of T1D (8) is largely mediated by CD4+CD25+ regulatory $\mathrm{T}$ lymphocytes $\mathrm{T}_{\text {reg }}$, which suppress the activity of effector $\mathrm{T}$ cells carrying destructive capacity. A decrease in $\mathrm{T}_{\text {reg }}$ spleen cells occurs in conjunction with an increased incidence of T1D in the BBDP rat (9), suggesting a potential role for these cells in the generation of T1D.

In this animal model, diet is postulated to be a primary environmental factor in promoting BBDP T1D (10). Before the onset of diabetes, the BBDP rat, in comparison with its nondiabetes prone progenitor strain, exhibits greater intestinal permeability associated with low levels of the major intercellular tight junction protein claudin-1 (4). Previous studies of dietary triggers in rodent models of T1D have used animals that had been weaned from maternal milk to solid diets, so they may not accurately reflect nutrition during the neonatal period. The artificial rearing method for infant rats, usually called the pup-in-the-cup model, allows for specific nutritional intervention to individual rat pups during the suckling period (11). This technique of implanting an intragastric cannula allows for consistent access to the gastrointestinal tract and consequently the control and manipulation of both the composition and quantity of dietary intake in the preweaning rat pups.

We hypothesized that through the down-regulation of $\mathrm{T}_{\text {reg }}$ and anti-inflammatory cytokines, feeding with a formula (RMS) containing intact bovine proteins (versus exclusive maternal feeding) during the neonatal period would accelerate the development of T1D in BBDP rats. Testing this hypothesis is also critical as a first step for a series of proposed studies to evaluate dietary interventions for T1D using the pup-in-the-

\footnotetext{
Abbreviations: BBDP, diabetes-prone BioBreeding; CINC, cytokineinduced neutrophil chemoattractant; RMS, rat milk substitute; T1D, type 1 diabetes; $\mathbf{T}_{\text {reg }}, \mathrm{CD} 4+\mathrm{CD} 25+$ regulatory $\mathrm{T}$ lymphocytes
} 
cup model. Therefore, we compared the effects of formula feeding in the pup-in-the-cup model with the MF model on regulatory T-cell frequency, inflammatory mediators, and the rates of diabetes-free survival.

\section{METHODS}

Animals. All animal studies were approved by the Institutional Animal Care and Use Committee at the University of Florida. Pregnant BBDP/Wor rats were obtained from Biomedical Research Models, Inc. and housed in the Animal Care Services facility, where they delivered litters of six to 10 pups. At $7 \mathrm{~d}$ of age, pups were randomized to the exclusively MF model or to the pup-in-the-cup model feeding with a prepared formula, based on the composition of rat milk, but lacking host defense factors such as immunoglobulin and antibacterial peptides (rat milk substitute (RMS)) (12). Under isoflurane anesthesia, feeding tubes constructed from 14-cm sections of polyethylene tubing were inserted into the stomach as previously described (13). All pups underwent anesthesia and gastrostomy placement, but the MF group was returned to their mothers, and the RMS group received formula feeding. Timer-controlled syringe pumps were connected to the feeding tubes and were set to deliver RMS for the first $20 \mathrm{~min}$ of every hour at a weightdependent flow rate (14). After a 24-h acclimation period during which they received RMS diluted 1:1 with sterile water, pups were fed through d 14 and then returned to their mothers. All pups were weaned to standard rodent chow at $d 23$. In some experiments, animals were killed at the end of the pup-inthe-cup period (d 14), while in others, they were euthanized at approximately $75 \mathrm{~d}$ of age, before the onset of overt diabetes. Samples of blood, intestine (ileum), liver, pancreas, thymus, and spleen were collected for analysis.

RMS formula composition. Our adaptation of the RMS formula is described in Table 1.

Determination of diabetes-free survival. Rats (12 in each group) were kept in conventional housing that was maintained at constant temperature

Table 1. Composition of rat milk substitute

\begin{tabular}{|c|c|}
\hline Macronutrients & $\mathrm{g} / \mathrm{L}$ RMS \\
\hline Fat & 140 \\
\hline MCT oil & 53 \\
\hline Soy oil & 26 \\
\hline Corn oil & 41 \\
\hline Coconut oil & 20 \\
\hline Protein & 117 \\
\hline Casein & 70 \\
\hline Whey & 47 \\
\hline Carbohydrate & 35 \\
\hline Lactose & 35 \\
\hline \multicolumn{2}{|l|}{ Minerals } \\
\hline Calcium & 4.5 \\
\hline Phosphorus & 1.34 \\
\hline Potassium & 1.48 \\
\hline Chloride & 1.52 \\
\hline Magnesium & 0.22 \\
\hline Zinc & 0.045 \\
\hline Iron & 0.0092 \\
\hline Copper & 0.012 \\
\hline \multicolumn{2}{|l|}{ Vitamins } \\
\hline Riboflavin & 0.0093 \\
\hline Niacin & 0.0145 \\
\hline Pyridoxal & 0.0077 \\
\hline Inositol & 0.518 \\
\hline Teklad mix & 4 \\
\hline \multicolumn{2}{|l|}{ Amino acids } \\
\hline Arginine & 6.25 \\
\hline Glycine & 3.75 \\
\hline Taurine & 1.9 \\
\hline Picolinic acid & 0.25 \\
\hline \multicolumn{2}{|l|}{ Others } \\
\hline Carnitine & 0.0342 \\
\hline Creatine & 0.049 \\
\hline Ethanolamine & 0.07 \\
\hline
\end{tabular}

with a 12-h light/dark cycle, with free access to a commercially available rodent diet (Harlan Teklad, Indianapolis, IN) and water. Onset of diabetes was assessed starting at age $60 \mathrm{~d}$ by monitoring rats for polyuria, polydipsia, weight loss, and/or lethargy. Diabetes was diagnosed clinically using urinalysis reagent strips (Multistix 9 SG; Bayer Corp., Pittsburgh, PA) to measure urine concentrations of glucose and ketones. Rats with glucose concentration $\geq 1000 \mathrm{mg} / \mathrm{dL}$ and ketone concentration $\geq 40 \mathrm{mg} / \mathrm{dL}$ were considered diabetic and were summarily euthanized.

Flow cytometry. Single-cell suspensions from spleen and thymus sections collected from 75-d-old prediabetic BBDP rats (five in each group) were prepared in Hanks' azide buffer by passing through nylon mesh cell strainers (BD Falcon, San Jose, CA). Erythrocytes were lysed in ammonium chloride lysis buffer, washed in phosphate-buffered saline (PBS), and centrifuged at $1300 \mathrm{rpm}$ for $5 \mathrm{~min}$. Pellets were resuspended in PBS and cells were counted using a hemocytometer. All antibodies and reagents for cytometric analysis were purchased from BD Biosciences (San Jose, CA) except the anti-murine Foxp3 staining set, which was purchased from eBioscience (San Diego, CA). After staining for cell surface molecules (CD4 and CD25) for $15 \mathrm{~min}$ at room temperature, cells were washed and then fixed according to manufacturer's recommendations. Cells were then washed in $1 \times$ permeabilization buffer twice, and then stained with anti-Foxp3 antibody for $30 \mathrm{~min}\left(4^{\circ} \mathrm{C}\right)$. Flow cytometry was performed on a four-color BD FACSCalibur cytometer. FCS Express (De Novo Software, version 2.200.0023; Thornhill, ON) was used for analysis of cytometric data.

Cytokine analysis. Sections of liver and small intestine (ileum) were collected from 14-d-old pups (six in each group) and 75-d-old adult BBDP rats (12 in each group) and homogenized on ice in a lysis buffer containing protease inhibitors. Homogenates were centrifuged twice for $30 \mathrm{~min}$ at 15,000 $\mathrm{rpm}$ and $4^{\circ} \mathrm{C}$ to remove tissue debris. Protein concentrations were determined using a modified Bradford-Lowry assay (BioRad Inc., Hercules, CA). Tissue extracts were placed into a commercially available multiplex enzyme-linked immunoassay system (Lincoplex; Linco Research, St. Louis, MO) and concentrations of the following cytokines measured: chemokine-inducedneutrophil chemoattractant (CINC), TNF- $\alpha$, IFN- $\gamma$, IL-4, IL-10, and IL-18. Microtiter plates were read using a Luminex-100 LabMap system (Luminex Corp., Austin, TX). Cytokine concentrations were determined using SOFTmax PRO software (Molecular Devices, Sunnyvale, CA) with four-parameter data analysis.

Statistical analysis. Values are given as mean \pm SD of duplicate measurements. $t$ Tests were performed using SigmaStat software (SPSS Science, Chicago, IL), and differences among means were considered significant at $p<0.05$.

\section{RESULTS}

Diabetes-free survival. BBDP rats exhibited overt glycosuria and ketonuria between 90 and $114 \mathrm{~d}$ of age. RMS and MF animals developed diabetes at similar rates $(p=0.59)$. Both groups had similar diabetes-free survival curves (Fig. 1). There were no significant differences in time of onset of diabetes between the two groups.

Regulatory T-cell frequency. $\mathrm{T}_{\mathrm{reg}}$ : $\mathrm{T}$ lymphocyte ratios did not differ between BBDP rats that were MF versus formula fed

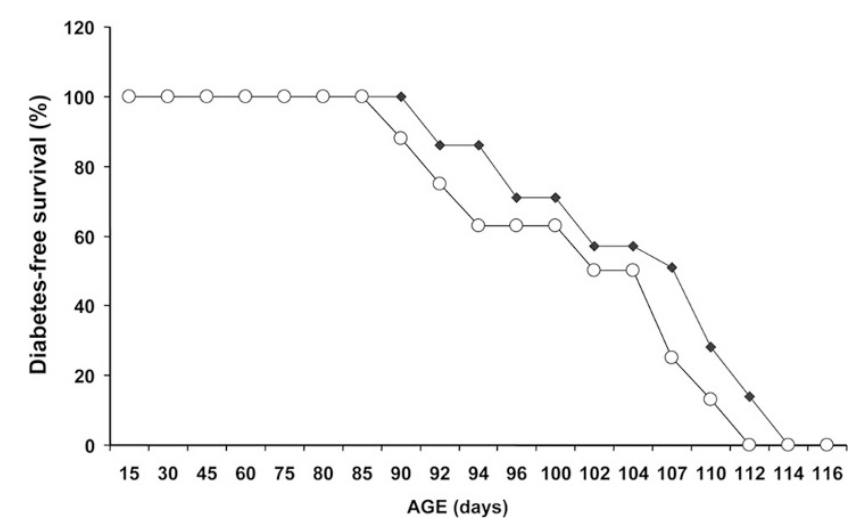

Figure 1. Diabetes-free survival curves in two populations of BBDP rats: RMS ( ) using the pup-in-the-cup system during the preweaning period $v s$ MF $(\bigcirc)$ exclusively during the preweaning period $(p=0.599)$. Rats with glucose concentration $\geq 1000 \mathrm{mg} / \mathrm{dL}$ and ketone concentration $\geq 40 \mathrm{mg} / \mathrm{dL}$ were considered diabetic and were summarily euthanized. 
Table 2. BBDP pups vs adults: ileum cytokine profiles

\begin{tabular}{|c|c|c|c|c|}
\hline \multirow[b]{3}{*}{ Cytokines (pg/mg protein) } & \multicolumn{4}{|c|}{ Ileum } \\
\hline & \multicolumn{2}{|c|}{$14 \mathrm{~d}$} & \multicolumn{2}{|c|}{$75 \mathrm{~d}$} \\
\hline & MF & RMS & MF & RMS \\
\hline CINC-1(GRO/KC) & $5.00 \pm 1.12$ & ND & $107.30 \pm 18.70 *$ & $70.40 \pm 50.80$ \\
\hline INF- $\gamma$ & $2.56 \pm 0.43$ & $2.57 \pm 0.42$ & $7.65 \pm 2.75^{*}$ & $7.26 \pm 4.85^{*}$ \\
\hline IL-10 & $1.79 \pm 0.44$ & $1.84 \pm 0.57$ & $30.54 \pm 8.21 *$ & $17.42 \pm 10.05^{*}$ \\
\hline IL-18 & $14.42 \pm 7.05 \dagger$ & $1.67 \pm 0.51$ & $225.23 \pm 88.04 *$ & $161.09 \pm 65.26^{*}$ \\
\hline IL-4 & ND & ND & $20.41 \pm 0.96$ & $19.48 \pm 0.57$ \\
\hline TNF- $\alpha$ & ND & ND & ND & ND \\
\hline
\end{tabular}

$\mathrm{ND}$, not detected.

$* p<0.01$ vs $14 \mathrm{~d}$.

$\dagger p<0.01$ vs RMS.

Table 3. BBDP pups vs adults: liver cytokine profiles

\begin{tabular}{|c|c|c|c|c|}
\hline \multirow{3}{*}{$\begin{array}{c}\text { Cytokines } \\
\text { (pg/mg protein) }\end{array}$} & \multicolumn{4}{|c|}{ Liver } \\
\hline & \multicolumn{2}{|c|}{$14 \mathrm{~d}$} & \multicolumn{2}{|c|}{$75 \mathrm{~d}$} \\
\hline & MF & RMS & MF & RMS \\
\hline CINC-1 (GRO/KC) & $4.60 \pm 0.83$ & $7.55 \pm 3.58$ & $134.84 \pm 55.75^{*}$ & $174.56 \pm 24.84^{*}$ \\
\hline INF- $\gamma$ & $1.08 \pm 0.26$ & $0.98 \pm 0.12$ & $17.22 \pm 2.11 *$ & $35.22 \pm 7.03^{*}$ \\
\hline IL-10 & $1.36 \pm 0.45$ & $1.38 \pm 0.27$ & $440.98 \pm 55.61^{*}$ & $263.18 \pm 35.08^{*}$ \\
\hline IL-18 & $237.05 \pm 58.89$ & $225.28 \pm 68.19$ & $29.00 \pm 4.24 *$ & $45.07 \pm 7.78^{*}$ \\
\hline IL-4 & ND & ND & $22.61 \pm 3.26^{*}$ & $29.59 \pm 1.91 *$ \\
\hline TNF- $\alpha$ & $6.41 \pm 1.51$ & $5.96 \pm 0.59$ & $18.59 \pm 3.35^{*}$ & $34.04 \pm 3.76^{*}$ \\
\hline
\end{tabular}

$* p<0.01$ vs $14 \mathrm{~d}$.

in spleen $(0.059 \pm 0.0044$ versus $0.0067 \pm 0.013, p=0.14)$ and in thymus $(0.0064 \pm 0.00054$ versus $0.0061 \pm 0.00033$ $p=0.79)$. In splenic samples, there were no significant differences between MF and RMS groups $(0.80 \pm 0.10$ versus $1.24 \pm 0.48, p=0.39$ ) in the frequency of regulatory $\mathrm{T}$ lymphocytes (CD4+/CD25+) expressing the foxp 3 transcription factor. CD4+/CD25+/foxp3-expressing T-cell numbers in thymic samples were likewise similar $(0.21 \pm 0.13$ versus $0.24 \pm 0.08, p=0.84$ ) in MF and RMS animals.

Cytokine profiles: comparison (pups versus adults). Concentrations of CINC, IFN- $\gamma$, IL-18, IL-4, and IL-10 were greater in ileal homogenates from 75-d-old adult rats than in those from 14-d-old pups, irrespective of neonatal feeding (Table 2). TNF- $\alpha$ was not detected in the ileal samples (Table 2). Adult liver homogenates had greater concentrations of CINC, IFN- $\gamma$, IL-4, and IL-10 than those from pups (Table 3).

Infant rats. The 14-d-old BBDP MF rat pups had significantly greater distal small intestinal concentrations of CINC $(p<0.001)$ and IL-18 $(p=0.002)$ than formula-fed pups (Fig. 2 and Table 2). Liver homogenates from both groups of pups showed no significant differences in cytokine concentrations (Table 3).

Prediabetic adult rats. Liver homogenates from RMS-fed 75-d-old animals (Fig. 3 and Table 3 ) had greater concentrations of TNF- $\alpha(p<0.001)$, IFN- $\gamma(p=0.002)$, IL-18 $(p=0.01)$, and $\mathrm{IL}-4(p=0.01)$ in comparison with MF controls. RMS liver samples had lower levels of IL-10 $(p=0.002)$. There were no significant differences in any of the measured cytokines in intestinal homogenates from the adult rats (Table 2).

\section{DISCUSSION}

Studies in monozygotic twins suggest that T1D is only minimally pairwise concordant, implying that there is either acquired postconceptional genetic discordance or differential environmental exposure (15). The 30 -fold greater T1D rate in Finland versus Japan (16) and the increase in T1D in a Polynesian cohort moving to industrialized New Zealand (15) suggest that genetic differences alone are not operative in this process. Additionally, the considerable increase in incidence of T1D globally over the past three decades is most likely not due to enhanced genetic disease susceptibility in the population, but rather reflects changes in lifestyle and environment

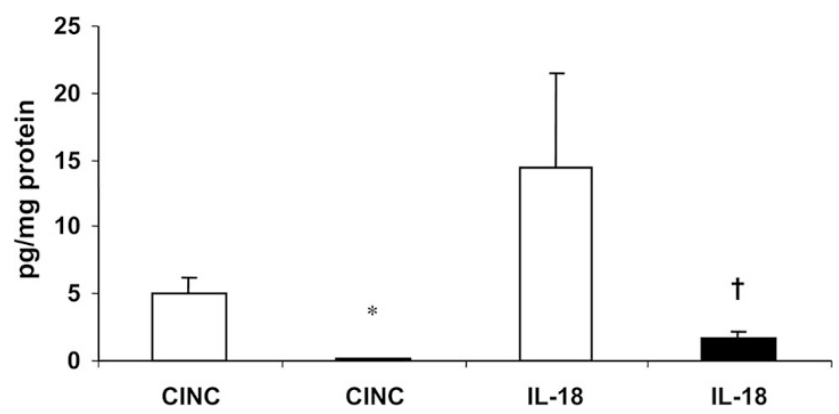

Figure 2. Concentrations of proinflammatory mediators determined by multiplex ELISA in homogenates of ileum (distal small intestine) collected from two groups of 14-d-old BBDP rat pups: exclusively MF ( $\square$ ) during the preweaning period vs RMS fed ( $\square$ ) using the pup-in-the-cup system during the preweaning period. CINC is the rodent equivalent of IL-8. $* p<0.001 v s$ MF; $\S p=0.002$ vs MF. 




Figure 3. Concentrations of cytokines determined by multiplex ELISA in homogenates of liver collected from 75-d-old prediabetic BBDP rats, MF ( $\square$ ), RMS fed ( $\square$ ) during the preweaning period. $* p<0.001, * * p=0.002 ; \uparrow p=$ 0.01 vs MF.

(17). Therefore, intense investigation is being aimed toward defining the role of environmental triggers, which may provide targets for preventive and therapeutic measures.

One such putative environmental trigger is dietary antigens, particularly cow's milk proteins, which in the industrialized world are usually the first foreign antigens encountered by the neonatal gut. In population-based studies, a relationship between consumption of cow's milk proteins and the incidence of T1D has been demonstrated. An odds ratio of 11.3, indicating a strong association, was found for cow's milk protein exposure in a group of children with T1D when compared with nondiabetic controls with the same high-risk HLA marker (18). In 1994, the American Academy of Pediatrics recommended exclusive breast-feeding and avoidance of intact cow's milk protein in the first year of life for infants with first-degree relatives having T1D (19). The prospective multicenter Trial to Reduce Insulin-Dependent Diabetes in the Genetically at Risk (TRIGR) is based on the hypothesis that avoidance of intact cow's milk protein feedings for at least the first 6 mo of life will reduce the incidence of T1D in a high-risk population (20). In this trial, significantly fewer (3.6\% versus $11.2 \%$ in the control group) subjects who during infancy received a formula with extensively hydrolyzed proteins (versus intact cow's milk proteins) developed at least one islet autoantibody at 2 y out (21). However, other prospective trials such as the Australian and German BabyDIAB studies have not shown a similar association between early exposure to cow's milk or duration of breastfeeding and development of markers of T1D, so this association remains a subject of controversy.

Similar to humans with T1D (22), the BBDP rat shows intestinal barrier dysfunction coupled with an early intestinal inflammatory response (23). The Canadian strain of diabetesprone $\mathrm{BB}$ rat (BBdp; note the different nomenclature) also has increased paracellular gastrointestinal permeability before the onset of diabetes, with the incidence of T1D reduced by feeding hydrolyzed rather than intact cow's milk proteins (23). In a separate study, BBdp rats receiving a diet in which hydrolyzed casein was the sole protein source had a reduction in expression of the disease (24). The above studies were conducted using animals that had been weaned from maternal milk to solid diets, so they may not accurately reflect nutrition during the neonatal period.
This study is the first to use the gastrostomy-based pup-in-thecup artificial feeding model to manipulate the neonatal diet in the BBDP rat, a genetically susceptible rodent model of type 1 diabetes. The aim was to test whether formula feeding during the preweaning period decreased the diabetes-free survival of this animal in comparison with exclusive maternal feeding. It is thought that the pathogenesis of T1D involves an autoimmune response triggered early in life by environmental antigens such as those found in a cow's milk protein-based formula. Through a more permeable neonatal intestinal barrier, these antigens more readily access the mucosal immune system, inducing a proinflammatory state and inhibiting the suppression of cytotoxic $\mathrm{T}$ lymphocytes, which eventually destroy pancreatic islet beta cells. In addition, maternal milk contains host defense factors such as antibacterial peptides, lysozyme, and secretory immunoglobulins not found in the RMS.

The pup-in-the-cup system provides the advantage of complete control of dietary content and quantity. However, it has several limitations. Pups weighing less than $10 \mathrm{~g}$ (about 6-7 $\mathrm{d}$ of age) are unlikely to survive the anesthesia and esophageal placement of gastric cannulae. Therefore, all pups in the study were exclusively MF for the first $7 \mathrm{~d}$ of life. To control for stress induced by anesthesia and surgery, all pups underwent the same anesthesia and instrumentation, but the gastric cannulae were left in place in the RMS-fed pups only. In our study, maternal separation during the $7 \mathrm{~d}$ of RMS feeding was necessary. The RMS-fed pups were stimulated twice daily with a cotton-tipped applicator to clean their coats and induce urination and defecation, and their litter was changed daily, partially simulating the maternal behavior of cleaning and stimulating the pups.

Previous studies have shown that separating rat pups from their mothers over a period of $2 \mathrm{wk}$ has been shown to induce altered intestinal permeability, especially in the colon, and altered cytokine mRNA expression (25). This study demonstrated increased levels of proinflammatory cytokines at 12 wk, whereas in our study, only the anti-inflammatory cytokine IL-10 was different and was elevated in the MF group. Thus, the pattern of cytokine elevation was not that which has previously been shown to be associated by the stress of maternal separation.

Our findings indicate that formula feeding does not accelerate the development of T1D in the BBDP rat. It does not affect the frequency and relative proportion of regulatory $\mathrm{T}$ lymphocytes, which keep autoimmune responses in check. BBDP rats fed RMS during the preweaning period did have lower intestinal concentrations of two prominent proinflammatory cytokines, IL-18 and CINC (GRO/KC), but these differences were not observed in intestine from adult animals. The hepatic cytokine profile was altered in adulthood as RMS-fed animals had higher levels of key proinflammatory mediators, TNF, IFN, and IL-18 with significantly lower levels of IL-10, an anti-inflammatory cytokine, than MF animals. Again, the differences in the proinflammatory cytokines between the two groups in the liver were augmented in the adult stage, before the onset of overt T1D. Despite the absence of differences in T1D incidence and $\mathrm{T}_{\text {reg }}$ frequencies, neonatal diet has a programming effect on the secretion of inflamma- 
tory mediators. The exception here was hepatic IL-18, which decreased between the pup and adult stages.

Likewise, we observed that exclusive maternal milk feeding of pups does not prevent or delay diabetes in the BBDP rat. It is more difficult, in general, to prevent diabetes in the BBDP rat than in the other major rodent model of T1D, the NOD mouse (26). The BBDP rat model is more stringently programmed to develop diabetes than the NOD mouse, so the neonatal diet may not have sufficed to alter the immunopathogenesis and final outcome. We did, however, observe "fingerprints" of a dietinduced effect on the mucosal immune system that was sustained and even augmented into the adult, prediabetic stage.

Of note is that in separate experiments, an attempt was made to compare the effects of feeding a hydrolyzed protein formula with whole proteins at the same concentrations using the pup-in-the-cup model to mimic the TRIGR trial. Interestingly, most of the hydrolyzed protein-fed pups died within 3 $\mathrm{d}$ of initiating the feeding. We surmised that the reason for the high mortality was the high osmolality $(>1200 \mathrm{mOsm} / \mathrm{kg})$ of the protein hydrolysate formula, which was needed to provide the relatively high quantity of protein required for growth in rat pups (much higher than that required by human infants).

In summary, the findings indicate that in this model of T1D, formula feeding during the neonatal period confers no disadvantage in terms of the natural history of the disease, nor does it alter the expression of regulatory $\mathrm{T}$ lymphocytes, mediators of oral tolerance. It does, however, "program" the mucosal inflammatory response into adulthood. Formula feeding is associated with lower hepatic levels of key proinflammatory cytokines and increased anti-inflammatory cytokine IL-10. This study is the first to examine neonatal diet in and apply the pup-in-the cup feeding system to the BBDP rat model of T1D. As both MF and RMS pups received anesthesia and a gastrostomy, it excludes the artificial feeding system as a trigger of the T1D process. We have thus established a baseline neonatal dietary intervention for future investigations, which may involve the use of probiotics, anti-inflammatory polyunsaturated fatty acid blends, or butyrate in neonatal rodent models in an effort to prevent the intestinal barrier dysfunction and loss of oral tolerance that leads to T1D.

\section{REFERENCES}

1. Vaarala O 2002 The gut immune system and type 1 diabetes. Ann N Y Acad Sci 958:39-46

2. Leslie RD, Elliott RB 1994 Early environmental events as a cause of IDDM. Evidence and implications. Diabetes 43:843-850

3. Meddings JB, Jarand J, Urbanski SJ, Hardin J, Gall DG 1999 Increased gastrointestinal permeability is an early lesion in the spontaneously diabetic BB rat. Am J Physiol 276:G951-G957
4. Neu J, Reverte CM, Mackey AD, Liboni K, Tuhacek-Tenace LM, Hatch M, Li N, Caicedo RA, Schatz DA, Atkinson M 2005 Changes in intestinal morphology and permeability in the biobreeding rat before the onset of type 1 diabetes. J Pediatr Gastroenterol Nutr 40:589-595

5. Vaarala O 1999 Gut and the induction of immune tolerance in type 1 diabetes. Diabetes Metab Res Rev 15:353-361

6. Secondulfo M, Iafusco D, Carratù R, deMagistris L, Sapone A, Generoso M Mezzogiomo A, Sasso FC, Cartenì M, De Rosa R, Prisco F, Esposito V 2004 Ultrastructural mucosal alterations and increased intestinal permeability in nonceliac, type I diabetic patients. Dig Liver Dis 36:35-45

7. Crisa L, Mordes JP, Rossini AA 1992 Autoimmune diabetes mellitus in the BB rat Diabetes Metab Rev 8:4-37

8. Rossini AA 2004 Autoimmune diabetes and the circle of tolerance. Diabetes 53:267-275

9. Poussier P, Ning T, Murphy T, Dabrowski D, Ramanathan S 2005 Impaired post-thymic development of regulatory $\mathrm{CD} 4+25+\mathrm{T}$ cells contributes to diabetes pathogenesis in BB rats. J Immunol 174:4081-4089

10. Malaisse WJ, Olivares E, Laghmich A, Ladriere L, Sener A, Scott FW 2000 Feeding a protective hydrolysed casein diet to young diabetic-prone BB rats affects oxidation of L[U-14C]glutamine in islets and Peyer's patches, reduces abnormally high mitotic activity in mesenteric lymph nodes, enhances islet insulin and tends to normalize NO production. Int J Exp Diabetes Res 1:121-130

11. Hall WG 1975 Weaning and growth of artificially-reared rats. Science 190:13131315

12. Auestad N, Korsak RA, Bergstrom JD, Edmond J 1989 Milk-substitutes comparable to rat's milk; their preparation, composition, and impact on development and metabolism in the artificially reared rat. Br J Nutr 61:495-518

13. Potsic B, Holliday N, Lewis P, Samuelson D, DeMarco V, Neu J 2002 Glutamine supplementation and deprivation: effect on artificially reared rat small intestinal morphology. Pediatr Res 52:430-436

14. Patel MR, DeMarco VG, Clare-Salzer M, Li YY, Neu J 2002 Indomethacin, dexamethasone, and intestinal damage in infant rats. J Pediatr Gastroenterol Nutr 35:154-161

15. Åkerblom HK, Vaarala O, Hyöty H, Ilonen J, Knip M 2002 Environmental factors in the etiology of type 1 diabetes. Am J Med Genet 115:18-29

16. LaPorte RE, Tajima N, Akerblom HK, Berlin N, Brosseau J, Christy M, Drash AL, Fishbein H, Green A, Hamman R 1985 Geographic differences in the risk of insulin-dependent diabetes mellitus: the importance of registries. Diabetes Care 8:101-107

17. Atkinson MA, Eisenbarth GS 2001 Type 1 diabetes: new perspectives on disease pathogenesis and treatment. Lancet 358:221-229

18. Kostraba JN, Cruickshanks KJ, Lawler-Heavner J, Jobim LF, Rewers MJ, Gay EC, Chase HP, Klingensmith G, Hamman RF 1993 Early exposure to cow's milk and solid foods in infancy, genetic predisposition, and risk of IDDM. Diabetes 42:288295

19. 1994 Infant feeding practices and their possible relationship to the etiology of diabetes mellitus. American Academy of Pediatrics Work Group on Cow's Milk Protein and Diabetes Mellitus. Pediatrics 94:752-754

20. Paronen J, Knip M, Savilahti E, Virtanen SM, Ilonen J, Akerblom HK, Vaarala O 2000 Effect of cow's milk exposure and maternal type 1 diabetes on cellular and humoral immunization to dietary insulin in infants at genetic risk for type 1 diabetes. Finnish Trial to Reduce IDDM in the Genetically at Risk Study Group. Diabetes 49:1657-1665

21. Wilson DM, Buckingham B 2001 Prevention of type 1a diabetes mellitus. Pediatr Diabetes 2:17-24

22. Westerholm-Ormio M, Vaarala O, Pihkala P, Ilonen J, Savilahti E 2003 Immunologic activity in the small intestinal mucosa of pediatric patients with type 1 diabetes. Diabetes 52:2287-2295

23. Hardin JA, Donegan L, Woodman RC, Trevenen C, Gall DG 2002 Mucosal inflammation in a genetic model of spontaneous type I diabetes mellitus. Can J Physiol Pharmacol 80:1064-1070

24. Scott FW, Rowsell P, Wang GS, Burghardt K, Kolb H, Flohe S 2002 Oral exposure to diabetes-promoting food or immunomodulators in neonates alters gut cytokines and diabetes. Diabetes 51:73-78

25. Barreau F, Ferrier L, Fioramonti J, Bueno L 2004 Neonatal maternal deprivation triggers long term alterations in colonic epithelial barrier and mucosal immunity in rats. Gut 53:501-506

26. Rossini AA, Handler ES, Mordes JP, Greiner DL 1995 Human autoimmune diabetes mellitus: lessons from BB rats and NOD mice-caveat emptor. Clin Immunol Immunopathol 74:2-9 